sciendo

\title{
DISTRIBUION OF LEADING DIGITS OF NUMBERS II
}

\author{
Yukio Ohkubo $^{1}$ - Oto Strauch ${ }^{2}$ \\ ${ }^{1}$ Dept. of Businessv Administration, The International University of Kagoshima, Kagoshima, JAPAN \\ ${ }^{2}$ Institue of Mathematics, Slovak Academy of Sciences, Bratislava, SLOVAKIA
}

\begin{abstract}
In this paper, we study the sequence $\left(f\left(p_{n}\right)\right)_{n \geq 1}$, where $p_{n}$ is the $n$th prime number and $f$ is a function of a class of slowly increasing functions including $f(x)=\log _{b} x^{r}$ and $f(x)=\log _{b}(x \log x)^{r}$, where $b \geq 2$ is an integer and $r>0$ is a real number. We give upper bounds of the discrepancy $D_{N_{i}}^{*}\left(f\left(p_{n}\right), g\right)$ for a distribution function $g$ and a sub-sequence $\left(N_{i}\right)_{i>1}$ of the natural numbers. Especially for $f(x)=\log _{b} x^{r}$, we obtain the effective results for an upper bound of $D_{N_{i}}^{*}\left(f\left(p_{n}\right), g\right)$.
\end{abstract}

\section{Communicated by Werner Georg Nowak}

\section{Introduction}

In [8], we gave some results about the first digit problem in base $b \geq 2$ for the sequence $\left(n^{r}\right)_{n \geq 1}$ and for the sequence $\left(p_{n}^{r}\right)_{n \geq 1}$, where $p_{n}$ is the $n$th prime number and $r>0$ is a real number. In this paper, we generalize and sharpen them.

Throughout this paper, for $x \in \mathbb{R}$, let

$$
\lfloor x\rfloor=\max \{n \in \mathbb{Z}: n \leq x\}, \quad\lceil x\rceil=\min \{n \in \mathbb{Z}: x \leq n\}, \quad\{x\}=x-\lfloor x\rfloor,
$$

let $c_{E}$ denote the characteristic function of the set $E \subset \mathbb{R}$, and let $\pi(x)$ be the number of primes not exceeding $x$.

(C) 2019 BOKU-University of Natural Resources and Life Sciences and Mathematical Institute, Slovak Academy of Sciences.

2010 Mathematics Subject Classification: 11K06, 11K31, 11K38.

Keyw ords: prime numbers, Benford's law, distribution function, discrepancy, regularly varying function.

${ }^{2}$ Supported by Vega Project 2/0109/18.

Licensed under the Creative Commons Atribution-NC-ND 4.0 International Public License. 


\section{YUKIO OHKUBO — OTO STRAUCH}

Let $\left(y_{n}\right)_{n \geq 1}$ be a sequence of real numbers. If there exists a strictly increasing sequence of positive integers $\left(N_{i}\right)_{i \geq 1}$ such that

$$
\lim _{i \rightarrow \infty} \frac{1}{N_{i}} \sum_{n=1}^{N_{i}} c_{[0, x)}\left(\left\{y_{n}\right\}\right)=g(x) \text { for every } x \in[0,1],
$$

then $g$ is called a distribution functions (d.f.) $\bmod 1$ of $\left(y_{n}\right)_{n \geq 1}$. Let $G\left(y_{n} \bmod 1\right)$ be the set of all d.f.'s of $\left(y_{n}\right)_{n \geq 1}$ (see [12]). If $G\left(y_{n} \bmod 1\right)=\{g(x)=x\}$, then the sequence $\left(y_{n}\right)_{n \geq 1}$ is said to be uniformly distributed mod1 (abbreviating u.d.mod 1$)$.

Suppose that $g$ is a non-decreasing function on $[0,1]$ with $g(0)=0, g(1)=1$. Then the extremal discrepancy $D_{N}\left(y_{n}, g\right)$ and the star discrepancy $D_{N}^{*}\left(y_{n}, g\right)$ of $\left(y_{n}\right)_{n \geq 1}$ with respect to $g$ are defined by

$$
D_{N}\left(y_{n}, g\right)=\sup _{0 \leq \alpha<\beta \leq 1}\left|\frac{1}{N} \sum_{n=1}^{N} c_{[\alpha, \beta)}\left(\left\{y_{n}\right\}\right)-(g(\beta)-g(\alpha))\right|
$$

and

$$
D_{N}^{*}\left(y_{n}, g\right)=\sup _{x \in[0,1]}\left|\frac{1}{N} \sum_{n=1}^{N} c_{[0, x)}\left(\left\{y_{n}\right\}\right)-g(x)\right|,
$$

respectively (see [12, 1.10.1]). If $g(x)=x$ for $0 \leq x \leq 1$, then $g$ is omitted in $D_{N}^{*}\left(y_{n}, g\right)$ or $D_{N}\left(y_{n}, g\right)$.

Let $b \geq 2$ be an integer considered as a base for the development of a real number $x>0$ and $M_{b}(x)$ be the mantissa of $x$ defined by $x=M_{b}(x) \times b^{n(x)}$ such that $1 \leq M_{b}(x)<b$ holds, where $n(x)$ is a uniquely determined integer. Let $D=d_{1} d_{2} \cdots d_{s}$ be a positive integer expressed in the base $b$, that is

$$
D=d_{1} b^{s-1}+d_{2} b^{s-2}+\cdots+d_{s-1} b+d_{s},
$$

where $d_{1} \neq 0$ and at the same time $D=d_{1} d_{2} \cdots d_{s}$ is considered as an $s$-consecutive block of digits in the base $b$. Note that for $x$ of the type $x=0.00 \cdots 0 d_{1} d_{2} \cdots d_{s} \cdots, d_{1}>0$, we have $M_{b}(x)=d_{1} \cdot d_{2} \cdots d_{s} \cdots$ and the first zero digits are omitted. Thus a positive real number $x$ has the first $s$-digits, starting a non-zero digit, that equal to $D=d_{1} d_{2} \cdots d_{s}$ if and only if

$$
\frac{D}{b^{s-1}}=d_{1} \cdot d_{2} \cdots d_{s} \leq M_{b}(x)<d_{1} \cdot d_{2} \cdots\left(d_{s}+1\right)=\frac{D+1}{b^{s-1}} .
$$

Let $\left(x_{n}\right)$ be a sequence of positive number, and let $F\left(D, s, N, x_{n}\right)$ be the number of integers $1 \leq n \leq N$ such that the leading block of $s$ digits (beginning with $\neq 0$ ) of $x_{n}$ equals $D$. The sequence $\left(x_{n}\right)_{n \geq 1}$ is said to satisfy Benford's law (abbreviated by B. L.) in base $b$, if for every $s=1,2, \ldots$ and every $s$-digits integer $D=d_{1} d_{2} \cdots d_{s}$ 


\section{DISTRIBUTION OF LEADING DIGITS OF NUMBERS II}

$$
\lim _{N \rightarrow \infty} \frac{F\left(D, s, N, x_{n}\right)}{N}=\log _{b}\left(\frac{D+1}{b^{s-1}}\right)-\log _{b}\left(\frac{D}{b^{s-1}}\right)
$$

(see [3]). Since $\log _{b} M_{b}(x)=\left\{\log _{b} x\right\}$ by (1), the leading block of $s$ digits (beginning with $\neq 0$ ) of $x_{n}$ equals $D$ if and only if

$$
\log _{b}\left(\frac{D}{b^{s-1}}\right) \leq\left\{\log _{b} x_{n}\right\}<\log _{b}\left(\frac{D+1}{b^{s-1}}\right) .
$$

Hence, the sequence $\left(x_{n}\right)_{n \geq 1}$ satisfies Benford's law if and only if $\left(\log _{b} x_{n}\right)_{n \geq 1}$ is uniformly distributed mod 1 (see [3], 9]).

If $g \in G\left(\log _{b} x_{n} \bmod 1\right)$ and

$$
\lim _{i \rightarrow \infty} \frac{1}{N_{i}} \sum_{n=1}^{N_{i}} c_{[0, x)}\left(\left\{\log _{b} x_{n}\right\}\right)=g(x)
$$

for $x \in[0,1]$, then for every $s$-digits integer $D=d_{1} d_{2} \cdots d_{s}, s=1,2, \ldots$,

$$
\lim _{i \rightarrow \infty} \frac{F\left(D, s, N_{i}, x_{n}\right)}{N_{i}}=g\left(\log _{b}\left(\frac{D+1}{b^{s-1}}\right)\right)-g\left(\log _{b}\left(\frac{D}{b^{s-1}}\right)\right)
$$

(see [9, 8]).

For $r>0$, the sequence $\left(n^{r}\right)_{n \geq 1}$ and $\left(p_{n}^{r}\right)_{n \geq 1}$ do not satisfy Benford's law, that is, the sequence $\left(\log _{b} n^{r}\right)_{n \geq 1}$ and $\left(\log _{b} p_{n}^{r}\right)_{n \geq 1}$ are not u.d. $\bmod 1$. Eliahou,Massé, and Schneider [4 showed that for a positive integer $r$, the discrepancy of the sequence $\left(y_{n}\right)=\left(\log _{10} n^{r}\right)_{n \geq 1},\left(\log _{10}(n \log n)^{r}\right)_{n \geq 1}$, or $\left(\log _{10} p_{n}^{r}\right)_{n \geq 1}$ satisfies $D_{\phi(r)}\left(y_{n}\right)=O\left(r^{-1}\right)$ with $\phi(r)=\left\lfloor e^{r}\right\rfloor$. Thus, when $r \rightarrow \infty$, these sequences satisfy Benford's law in a sense.

It is also known that

$$
G\left(\log _{b} n^{r} \bmod 1\right)=G\left(\log _{b} p_{n}^{r} \bmod 1\right)=\left\{g_{w}(x) ; 0 \leq w \leq 1\right\},
$$

where

$$
g_{w}(x)=\frac{1}{b^{\frac{w}{r}}} \frac{b^{\frac{x}{r}}-1}{b^{\frac{1}{r}}-1}+\frac{\min \left(b^{\frac{x}{r}}, b^{\frac{w}{r}}\right)-1}{b^{\frac{w}{r}}} \quad(0 \leq x \leq 1),
$$

and if $\lim _{i \rightarrow \infty}\left\{\log _{b} p_{N_{i}}^{r}\right\}=w$, then for $x \in[0,1]$

$$
\lim _{i \rightarrow \infty} \frac{1}{N_{i}} \sum_{n=1}^{N_{i}} c_{[0, x)}\left(\left\{\log _{b} p_{n}^{r}\right\}\right)=g_{w}(x)
$$

(see [13, [6], 12, 2.12.1], and [7), and so

$$
\lim _{i \rightarrow \infty} \frac{F\left(D, s, N_{i}, p_{n}^{r}\right)}{N_{i}}=g_{w}\left(\log _{b}\left(\frac{D+1}{b^{s-1}}\right)\right)-g_{w}\left(\log _{b}\left(\frac{D}{b^{s-1}}\right)\right) .
$$




\section{YUKIO OHKUBO - OTO STRAUCH}

As examples of $N_{i}$ and $w$, in [8] we gave the following:

- if $0<w<1$ and $N_{i}=\pi\left(b^{\frac{i+w}{r}}\right)$, then $\lim _{i \rightarrow \infty}\left\{\log _{b} p_{N_{i}}^{r}\right\}=w$,

- if $N_{i}=\pi\left(b^{\frac{i}{r}}\right)$, then $\lim _{i \rightarrow \infty}\left\{\log _{b} p_{N_{i}}^{r}\right\}=1$.

In [8], for rate of convergence of (2) we also proved that

$$
\left|\frac{F\left(D, s, N_{i}, p_{n}^{r}\right)}{N_{i}}-\frac{\left(\frac{D+1}{b^{s-1}}\right)^{\frac{1}{r}}-\left(\frac{D}{b^{s-1}}\right)^{\frac{1}{r}}}{b^{\frac{1}{r}}-1}\right| \leq C_{b, r}\left(\frac{1}{\log p_{N_{i}}}\right),
$$

for $N_{i}=\pi\left(b^{\frac{i}{r}}\right), i=1,2, \ldots$ and a constant $C_{b, r}>0$ depending on $b$ and $r$.

In this paper, to refine (3), we consider a generalized sequence $\left(f\left(p_{n}\right)\right)_{n \geq 1}$, where $f$ is a function of a class of slowly increasing functions including

Since

$$
f(x)=\log _{b} x^{r} \quad \text { and } \quad f(x)=\log _{b}(x \log x)^{r} .
$$

$$
\left|\frac{F\left(D, s, N, x_{n}\right)}{N}-\left(g\left(\log _{b} \frac{D+1}{b^{s-1}}\right)-g\left(\log _{b} \frac{D}{b^{s-1}}\right)\right)\right| \leq 2 D_{N}^{*}\left(\log _{b} x_{n}, g\right),
$$

an upper bound of $D_{N_{i}}^{*}\left(\log _{b} x_{n}, g\right)$ is the rate of convergence of $\frac{F\left(D, s, N_{i}, x_{n}\right)}{N_{i}}$. Especially, we obtain an effective result for an upper bound of $D_{N}^{*}\left(\log _{b} p_{n}^{r}, g\right)$.

\section{Preliminaries}

To characterize the class of slowly increasing functions, we need some definitions and results concerning regular variation.

Definition 1 ([2], p.18). A function $f$ is said to be regularly varying of index $\rho$ if it is real-valued, positive and measurable on $\left[t_{0}, \infty\right)$ for some $t_{0}>0$, and if

$$
\lim _{t \rightarrow \infty} \frac{f(\lambda t)}{f(t)}=\lambda^{\rho} \quad(\forall \lambda>0)
$$

for some real number $\rho$. Then we write $f \in R_{\rho}$. If $f \in R_{0}$, then $f$ is said to be slowly varying.

LEMma 1 ([2], Theorem 1.3.1). The function $\ell$ is slowly varying if and only if it may be written in the form

$$
\ell(x)=c(x) \exp \left\{\int_{a}^{x} \varepsilon(u) \mathrm{d} u / u\right\} \quad(x \geq a)
$$

for some $a>0$, where $c(x)$ is measurable and $c(x) \rightarrow c \in(0, \infty), \varepsilon(x) \rightarrow 0$ as $x \rightarrow \infty$. 


\section{DISTRIBUTION OF LEADING DIGITS OF NUMBERS II}

Lemma 2. If a function $f$ is positive, strictly increasing, differentiable on $\left[t_{0}, \infty\right)$ for some $t_{0}>0$, and satisfies

$$
\lim _{t \rightarrow \infty} \frac{t f^{\prime}(t)}{f(t)}=\rho \neq 0,
$$

then $f \in R_{\rho}$ and $f^{-1} \in R_{1 / \rho}$.

Pro of. Let $\ell(t)=t^{-\rho} f(t)$ for $t \geq t_{0}$. Then we have

Since

$$
\varepsilon(t):=\frac{t \ell^{\prime}(t)}{\ell(t)}=-\rho+\frac{t f^{\prime}(t)}{f(t)} \rightarrow 0 \quad(t \rightarrow \infty) .
$$

$$
\int_{t_{0}}^{t} \frac{\varepsilon(u)}{u} \mathrm{~d} u=\int_{t_{0}}^{t} \frac{\ell^{\prime}(u)}{\ell(u)} \mathrm{d} u=\int_{t_{0}}^{t}(\log \ell(u))^{\prime} \mathrm{d} u=\log \ell(t)-\log \ell\left(t_{0}\right),
$$

we have

$$
\ell(t)=\ell\left(t_{0}\right) \exp \left(\int_{t_{0}}^{t} \frac{\varepsilon(u)}{u} \mathrm{~d} u\right) .
$$

From Lemma 1, it follows that $\ell(t)$ is slowly varying. Hence $f(t)=t^{\rho} \ell(t)$ is regularly varying of index $\rho$, i.e., $f \in R_{\rho}$.

Let $h(t)=\log f\left(e^{t}\right)$. Then

$$
h^{\prime}(t)=\frac{f^{\prime}\left(e^{t}\right) e^{t}}{f\left(e^{t}\right)}=\frac{\left\{\rho e^{(\rho-1) t} \ell\left(e^{t}\right)+e^{\rho t} \ell^{\prime}\left(e^{t}\right)\right\} e^{t}}{e^{\rho t} \ell\left(e^{t}\right)}=\rho+\frac{e^{t} \ell^{\prime}\left(e^{t}\right)}{\ell\left(e^{t}\right)}=\rho+\varepsilon\left(e^{t}\right) .
$$

Therefore by (4) we have

Hence

$$
h^{\prime}(t) \rightarrow \rho \quad(t \rightarrow \infty)
$$

$$
\left(h^{-1}\right)^{\prime}(t)=\frac{1}{h^{\prime}\left(h^{-1}(t)\right)} \rightarrow \frac{1}{\rho} \quad(t \rightarrow \infty) .
$$

Since $h^{-1}(t)=\log f^{-1}\left(e^{t}\right)$, we have

Hence

$$
f^{-1}(t)=\exp \left(h^{-1}(\log t)\right) .
$$

$$
\begin{aligned}
\frac{f^{-1}(\lambda t)}{f^{-1}(t)} & =\frac{\exp \left(h^{-1}(\log \lambda+\log t)\right)}{\exp \left(h^{-1}(\log t)\right)} \\
& =\exp \left\{h^{-1}(\log \lambda+\log t)-h^{-1}(\log t)\right\} \\
& =\exp \left\{(\log \lambda)\left(h^{-1}\right)^{\prime}\left(\xi_{t}\right)\right\},
\end{aligned}
$$

where $\log t \leq \xi_{t} \leq \log t+\log \lambda$. By this and (5) we have

$$
\frac{f^{-1}(\lambda t)}{f^{-1}(t)} \rightarrow \exp \left(\frac{1}{\rho} \log \lambda\right)=\lambda^{1 / \rho} \quad(t \rightarrow \infty) .
$$

Thus $f^{-1} \in R_{1 / \rho}$. 


\section{YUKIO OHKUBO - OTO STRAUCH}

Lemma 3 (Uniform Convergence Theorem: 2, [11 ). If $f \in R_{\rho}$, then for every $[a, b]$ with $0<a<b<\infty$

$$
\lim _{t \rightarrow \infty} \frac{f(\lambda t)}{f(t)}=\lambda^{\rho} \quad \text { uniformly in } \lambda \in[a, b] .
$$

LEMMA 4. If a real-valued function $f$ is strictly increasing and differentiable on $\left[t_{0}, \infty\right)$ for some $t_{0}>0$, and satisfies $\lim _{t \rightarrow \infty} t f^{\prime}(t)=\rho>0$, then for $-\infty<$ $a<b<\infty$

$$
\sup _{x \in[a, b]}\left|\frac{f^{-1}(t+x)}{f^{-1}(t)}-e^{x / \rho}\right| \rightarrow 0 \quad(t \rightarrow \infty) .
$$

P r o o f. Let $g(t)=\exp (f(t))$. From the assumption,

$$
\frac{t g^{\prime}(t)}{g(t)}=t f^{\prime}(t) \rightarrow \rho \quad(t \rightarrow \infty)
$$

By Lemma 2 we have $g^{-1} \in R_{1 / \rho}$. Since $g^{-1}(y)=f^{-1}(\log y)$, Lemma 3 implies for $\left[a^{\prime}, b^{\prime}\right]$ with $0<a^{\prime}<b^{\prime}<\infty$

$$
\lim _{y \rightarrow \infty} \frac{f^{-1}(\log (\lambda y))}{f^{-1}(\log y)}=\lambda^{1 / \rho} \quad \text { uniformly in } \lambda \in\left[a^{\prime}, b^{\prime}\right],
$$

and so

$$
\lim _{t \rightarrow \infty} \frac{f^{-1}(t+x)}{f^{-1}(t)}=e^{x / \rho} \quad(t \rightarrow \infty)
$$

uniformly for $x \in[a, b](-\infty<a<b<\infty)$, that is,

$$
\sup _{x \in[a, b]}\left|\frac{f^{-1}(t+x)}{f^{-1}(t)}-e^{x / \rho}\right| \rightarrow 0 \quad(t \rightarrow \infty) .
$$

LEMMA 5. If a real-valued function $f$ is strictly increasing, differentiable on $\left[t_{0}, \infty\right)$, and satisfies $\lim _{t \rightarrow \infty} t f^{\prime}(t)=\rho>0$, then for $-\infty<a<b<\infty$

$$
\sup _{x \in[a, b]}\left|\frac{B(t+x)}{B(t)}-e^{x / \rho}\right| \rightarrow 0 \quad(t \rightarrow \infty),
$$

where $B(t)=\frac{f^{-1}(t)}{\log f^{-1}(t)-1}$.

P r o of. Since $\lim _{t \rightarrow \infty} \frac{f(t)}{\log t}=\lim _{t \rightarrow \infty} t f^{\prime}(t)=\rho$, we have $\lim _{t \rightarrow \infty} f(t)=\infty$, and so $\lim _{t \rightarrow \infty} f^{-1}(t)=\infty$. By Lemma 4 we have

$$
\left|\frac{\log f^{-1}(t)-1}{\log f^{-1}(t+x)-1}-1\right|=\frac{\log \frac{f^{-1}(t+x)}{f^{-1}(t)}}{\log f^{-1}(t+x)-1} \leq \frac{\log \frac{f^{-1}(t+1)}{f^{-1}(t)}}{\log f^{-1}(t)-1} \rightarrow 0 \quad(t \rightarrow \infty)
$$

for $t \geq 0$ and $0 \leq x \leq 1$. 
Therefore,

$$
\begin{aligned}
& \sup _{x \in[a, b]}\left|\frac{B(t+x)}{B(t)}-e^{x / \rho}\right|=\sup _{0 \leq x \leq 1}\left|\frac{f^{-1}(t+x)}{f^{-1}(t)} \cdot \frac{\log f^{-1}(t)-1}{\log f^{-1}(t+x)-1}-e^{x / \rho}\right| \\
& \leq \sup _{0 \leq x \leq 1}\left(\frac{f^{-1}(t+x)}{f^{-1}(t)}\left|\frac{\log f^{-1}(t)-1}{\log f^{-1}(t+x)-1}-1\right|\right)+\sup _{0 \leq x \leq 1}\left|\frac{f^{-1}(t+x)}{f^{-1}(t)}-e^{x / \rho}\right| \\
& \leq \frac{f^{-1}(t+1)}{f^{-1}(t)} \sup _{0 \leq x \leq 1}\left|\frac{\log f^{-1}(t)-1}{\log f^{-1}(t+x)-1}-1\right|+\sup _{0 \leq x \leq 1}\left|\frac{f^{-1}(t+x)}{f^{-1}(t)}-e^{x / \rho}\right| \\
& \rightarrow 0 \quad(t \rightarrow \infty) .
\end{aligned}
$$

\section{Estimate of discrepancy of $\left(f\left(p_{n}\right)\right)$}

In [8], we obtained an upper bound of $D_{N}^{*}\left(\log _{b}\left(p_{n}^{r}\right), g_{w}\right)$ for $w \in[0,1]$. In slightly different fashion from [ 8 , we have the following estimate of $D_{N}^{*}\left(f\left(p_{n}\right), g_{w}\right)$ for $w \in[0,1]$, where $f$ is a strictly increasing continuous function on $[X, \infty)$ for some $X>0$.

TheOrem 1. Suppose that $f$ is a strictly increasing continuous function on $\left[p_{m+1}, \infty\right)$ for some integer $m \geq 0, k_{0}$ is an integer satisfying $f^{-1}\left(k_{0}\right)>$ $\max \left(p_{m+1}, e\right)$, and $\psi$ is an increasing positive function on $[0,1]$ with $\psi(0)=1$. Let

$$
B(x)=\frac{f^{-1}(x)}{\log f^{-1}(x)-1}, \quad K_{N}=\left\lfloor f\left(p_{N}\right)\right\rfloor, \quad \text { and } \quad w_{N}=\left\{f\left(p_{N}\right)\right\} .
$$

Then for $0 \leq w \leq 1$ the sequence $\left(f\left(p_{m+n}\right)\right)_{n \geq 1}, n=1,2, \ldots$ satisfies

$$
\begin{aligned}
& D_{N}^{*}\left(f\left(p_{m+n}\right), g_{w}\right) \leq \frac{2}{N} \sum_{k=k_{0}}^{K_{N}-1} B(k) \sup _{x \in[0,1]}\left|\frac{B(k+x)}{B(k)}-\psi(x)\right| \\
& +(\psi(1)+1) \frac{B\left(K_{N}\right)}{N} \sup _{x \in[0,1]}\left|\frac{B\left(K_{N}+x\right)}{B\left(K_{N}\right)}-\psi(x)\right| \\
& +\left(\psi(1) \frac{B\left(K_{N}\right)}{N}+1\right)\left|\psi(w)-\psi\left(w_{N}\right)\right|+\frac{B\left(k_{0}\right)}{N} \\
& +O\left(\frac{1}{N} \sum_{k=k_{0}+1}^{K_{N}+1} \frac{f^{-1}(k)}{\left(\log f^{-1}(k)\right)^{3}}\right)+O\left(\frac{\psi(1) B\left(K_{N}\right)}{N\left(\log f-1\left(K_{N}\right)\right)^{2}}\right) \\
& +O\left(\frac{f^{-1}\left(k_{0}\right)+m}{N}\right)+O\left(\frac{\psi(1) \log f^{-1}\left(K_{N}\right)}{N\left(\log f^{-1}\left(K_{N}\right)-1\right)}\right)
\end{aligned}
$$




\section{YUKIO OHKUBO - OTO STRAUCH}

where

$$
g_{w}(x)=\frac{1}{\psi(w)} \frac{\psi(x)-1}{\psi(1)-1}+\frac{\min (\psi(x), \psi(w))-1}{\psi(w)} \quad(0 \leq x \leq 1) .
$$

Proof. Set

$$
F_{N}(x)=\frac{\#\left\{1 \leq n \leq N ;\left\{f\left(p_{m+n}\right)\right\} \in[0, x)\right\}}{N} \quad \text { for } \quad x \in[0,1] .
$$

Then, for $0 \leq x \leq 1$

$$
\begin{aligned}
F_{N}(x)= & \frac{\#\left\{1 \leq n \leq N-m ;\left\{f\left(p_{m+n}\right)\right\} \in[0, x)\right\}}{N} \\
& +\frac{\#\left\{N-m+1 \leq n \leq N ;\left\{f\left(p_{m+n}\right)\right\} \in[0, x)\right\}}{N} \\
= & \frac{1}{N} \sum_{k=k_{0}}^{K_{N}-1}(A(k+x)-A(k)) \\
& +\frac{\min \left(A\left(K_{N}+x\right), A\left(K_{N}+w_{N}\right)\right)-A\left(K_{N}\right)}{N}+\frac{O\left(f^{-1}\left(k_{0}\right)+m\right)}{N},
\end{aligned}
$$

where

and

$$
A(y)=\#\left\{1 \leq n \leq N: f\left(p_{m+n}\right)<y\right\} \quad \text { for } y \geq f\left(p_{m+1}\right)
$$

$$
O\left(f^{-1}\left(k_{0}\right)+m\right) \leq f^{-1}\left(k_{0}\right)+m .
$$

Then, for $y \geq f\left(p_{m+1}\right)$

Since

$$
A(y)=\pi\left(f^{-1}(y)\right)-m+O(1) .
$$

(see [5]) and that

$$
\pi(x)=\int_{2}^{x} \frac{1}{\log t} \mathrm{~d} t+O\left(\frac{x}{(\log x)^{3}}\right)
$$

we have

$$
\int_{2}^{x} \frac{1}{\log t} \mathrm{~d} t=\frac{x}{\log x-1}+O\left(\frac{x}{(\log x)^{3}}\right),
$$

Therefore, for $y \geq f\left(p_{m+1}\right)$

$$
\pi(x)=\frac{x}{\log x-1}+O\left(\frac{x}{(\log x)^{3}}\right) .
$$

$$
A(y)=B(y)+O\left(\frac{f^{-1}(y)}{\left(\log f^{-1}(y)\right)^{3}}\right),
$$

and so,

$$
A(k+x)-A(k)=B(k+x)-B(k)+O\left(\frac{f^{-1}(k+1)}{\left(\log f^{-1}(k+1)\right)^{3}}\right)
$$

for $k \geq k_{0}$ and $0 \leq x \leq 1$. 
Since $B(x)$ and $f^{-1}(x) /\left(\log f^{-1}(x)\right)^{3}$ are non-decreasing, by (77) and (8) we have

$$
\begin{aligned}
& \min \left(A\left(K_{N}+x\right), A\left(K_{N}+w_{N}\right)\right)= \\
& \min \left(B\left(K_{N}+x\right), B\left(K_{N}+w_{N}\right)\right)+O\left(\frac{f^{-1}\left(K_{N}+1\right)}{\left(\log f^{-1}\left(K_{N}+1\right)\right)^{3}}\right) .
\end{aligned}
$$

Hence, by (6) and (8) we have

$$
\begin{array}{r}
F_{N}(x)=\frac{1}{N} \sum_{k=k_{0}}^{K_{N}-1}(B(k+x)-B(k))+\frac{\min \left(B\left(K_{N}+x\right), B\left(K_{N}+w_{N}\right)\right)-B\left(K_{N}\right)}{N}+ \\
O\left(\frac{1}{N} \sum_{k=k_{0}+1}^{K_{N}+1} \frac{f^{-1}(k)}{\left.\log f^{-1}(k)\right)^{3}}\right)+\frac{O\left(f^{-1}\left(k_{0}\right)+m\right)}{N} .
\end{array}
$$

We can express the first term in the right-hand side of (9) as

$$
\frac{\sum_{k=k_{0}}^{K_{N}-1}(B(k+x)-B(k))}{\sum_{k=k_{0}}^{K_{N}-1}(B(k+1)-B(k))} \cdot \frac{B\left(K_{N}\right)-B\left(k_{0}\right)}{N},
$$

and the second term as

$$
\left(\min \left(\frac{B\left(K_{N}+x\right)}{B\left(K_{N}\right)}, \frac{B\left(K_{N}+w_{N}\right)}{B\left(K_{N}\right)}\right)-1\right) \frac{B\left(K_{N}\right)}{N} .
$$

Hence we have for $x \in[0,1]$

$$
\begin{aligned}
F_{N}(x)-g_{w}(x) \\
=\frac{\sum_{k=k_{0}}^{K_{N}-1}(B(k+x)-B(k))}{\sum_{k=k_{0}}^{K_{N}-1}(B(k+1)-B(k))} \cdot \frac{B\left(K_{N}\right)-B\left(k_{0}\right)}{N}-\frac{\psi(x)-1}{\psi(1)-1} \cdot \frac{1}{\psi(w)} \\
+\left(\min \left(\frac{B\left(K_{N}+x\right)}{B\left(K_{N}\right)}, \frac{B\left(K_{N}+w_{N}\right)}{B\left(K_{N}\right)}\right)-1\right) \frac{B\left(K_{N}\right)}{N}-\frac{\min (\psi(x), \psi(w))-1}{\psi(w)} \\
+O\left(\frac{1}{N} \sum_{k=k_{0}+1}^{K_{N}+1} \frac{f^{-1}(k)}{\left.\log f^{-1}(k)\right)^{3}}\right)+\frac{O\left(f^{-1}\left(k_{0}\right)+m\right)}{N} \\
=\left(\frac{\sum_{k=k_{0}}^{K_{N}-1}(B(k+x)-B(k))}{\sum_{k=k_{0}}^{K_{N}-1}(B(k+1)-B(k))}-\frac{\psi(x)-1}{\psi(1)-1}\right) \frac{B\left(K_{N}\right)-B\left(k_{0}\right)}{N} \\
+\left(\frac{B\left(K_{N}\right)-B\left(k_{0}\right)}{N}-\frac{1}{\psi(w)}\right) \frac{\psi(x)-1}{\psi(1)-1} \\
+\left(\min \left(\frac{B\left(K_{N}+x\right)}{B\left(K_{N}\right)}, \frac{B\left(K_{N}+w_{N}\right)}{B\left(K_{N}\right)}-\min (\psi(x), \psi(w))\right) \frac{B\left(K_{N}\right)}{N}\right.
\end{aligned}
$$




$$
\begin{aligned}
& +\left(\psi(u)-\frac{N}{B\left(K_{N}\right)}\right)\left(\frac{\min (\psi(x), \psi(w))-1}{\frac{N}{B\left(K_{N}\right)} \psi(w)}\right) \\
& +O\left(\frac{1}{N} \sum_{k=k_{0}+1}^{K_{N}+1} \frac{f^{-1}(k)}{\left.\log f^{-1}(k)\right)^{3}}\right)+\frac{O\left(f^{-1}\left(k_{0}\right)+m\right)}{N}
\end{aligned}
$$

In order to estimate (11), we set

$$
S_{j}=\sum_{k=k_{0}}^{j-1}(B(k+x)-B(k)), \quad T_{j}=\sum_{k=k_{0}}^{j-1}(B(k+1)-B(k))
$$

for $j \geq k_{0}+1, S_{k_{0}}=T_{k_{0}}=0$, and $U=\frac{\psi(x)-1}{\psi(1)-1}$. Then we have, successively,

$$
\begin{aligned}
& \frac{\sum_{k=k_{0}}^{K_{N}-1}(B(k+x)-B(k))}{\sum_{k=k_{0}}^{K_{N}-1}(B(k+1)-B(k))}-\frac{\psi(x)-1}{\psi(1)-1}=\frac{S_{K_{N}}}{T_{K_{N}}}-U \\
& =\frac{1}{T_{K_{N}}} \sum_{k=k_{0}}^{K_{N}-1}\left(S_{k+1}-S_{k}\right)-\frac{U}{T_{K_{N}}} \sum_{k=k_{0}}^{K_{N}-1}\left(T_{k+1}-T_{k}\right) \\
& =\frac{1}{T_{K_{N}}} \sum_{k=k_{0}}^{K_{N}-1}\left(T_{k+1}-T_{k}\right)\left(\frac{S_{k+1}-S_{k}}{T_{k+1}-T_{k}}-U\right) \\
& =\frac{1}{B(K)-B\left(k_{0}\right)} \times \sum_{k=k_{0}}^{K_{N}-1}(B(k+1)-B(k))\left(\frac{B(k+x)-B(k)}{B(k+1)-B(k)}-\frac{\psi(x)-1}{\psi(1)-1}\right) .
\end{aligned}
$$

Then, we obtain

$$
\begin{aligned}
\mid \text { (11) } \mid \leq & \frac{1}{N} \sum_{k=k_{0}}^{K_{N}-1}(B(k+1)-B(k))\left|\frac{B(k+x)-B(k)}{B(k+1)-B(k)}-\frac{\psi(x)-1}{\psi(1)-1}\right| \\
= & \frac{1}{N} \sum_{k=k_{0}}^{K_{N}-1}(B(k+1)-B(k))\left|\frac{\Psi_{k}(x)-1}{\Psi_{k}(1)-1}-\frac{\psi(x)-1}{\psi(1)-1}\right| \\
\leq & \frac{1}{N} \sum_{k=k_{0}}^{K_{N}-1}(B(k+1)-B(k)) \\
& \times\left|\frac{\left(\Psi_{k}(x)-\psi(x)\right)(\psi(1)-1)-\left(\Psi_{k}(1)-\psi(1)\right)(\psi(1)-1)}{\left(\Psi_{k}(1)-1\right)(\psi(1)-1)}\right| \\
\leq & \frac{1}{N} \sum_{k=k_{0}}^{K_{N}-1} \frac{B(k+1)-B(k)}{\Psi_{k}(1)-1}\left(\left|\Psi_{k}(x)-\psi(x)\right|+\left|\Psi_{k}(1)-\psi(1)\right|\right)
\end{aligned}
$$




$$
\leq \frac{2}{N} \sum_{k=k_{0}}^{K_{N}-1} B(k) \sup _{x \in[0,1]}\left|\Psi_{k}(x)-\psi(x)\right|,
$$

where $\Psi_{k}(x)=\frac{B(k+x)}{B(k)}$.

Every second factor in (12), (13) and (14) is bonded. In fact, for (12)

Since

$$
0 \leq \frac{\psi(x)-1}{\psi(1)-1} \leq 1 \quad \text { for } x \in[0,1] .
$$

$$
N=A\left(K_{N}+w_{N}\right)+m+1=B\left(K_{N}+w_{N}\right)+O\left(\frac{f^{-1}\left(K_{N}+w_{N}\right)}{\left(\log f^{-1}\left(K_{N}+w_{N}\right)\right)^{3}}\right)+m+1,
$$

we have

$$
\frac{N}{B\left(K_{N}\right)}=\Psi_{K_{N}}\left(w_{N}\right)+R(N)
$$

where

$$
R(N)=O\left(\frac{1}{\left(\log f^{-1}\left(K_{N}\right)\right)^{2}}\right)+O\left(\frac{(m+1) \log f^{-1}\left(K_{N}\right)}{f^{-1}\left(K_{N}\right)}\right) \rightarrow 0 \quad(N \rightarrow \infty) .
$$

By (16), we have

$$
\begin{aligned}
|(12)| \leq & \left|\frac{B\left(K_{N}\right)-B\left(k_{0}\right)}{N}-\frac{1}{\psi(w)}\right| \\
\leq & \left|\frac{1}{\frac{N}{B\left(K_{N}\right)}}-\frac{1}{\psi\left(w_{N}\right)}\right|+\left|\frac{1}{\psi\left(w_{N}\right)}-\frac{1}{\psi(w)}\right|+\frac{B\left(k_{0}\right)}{N} \\
= & \left|\frac{1}{\Psi_{K_{N}}\left(w_{N}\right)+R(N)}-\frac{1}{\psi\left(w_{N}\right)}\right|+\frac{\left|\psi(w)-\psi\left(w_{N}\right)\right|}{\psi\left(w_{N}\right) \psi(w)}+\frac{B\left(k_{0}\right)}{N} \\
\leq & \frac{\psi\left(w_{N}\right)-\Psi_{K_{N}}\left(w_{N}\right) \mid+R(N)}{\psi\left(w_{N}\right)\left(\Psi_{K_{N}}\left(w_{N}\right)+R(N)\right)}+\left|\psi(w)-\psi\left(w_{N}\right)\right|+\frac{B\left(k_{0}\right)}{N} \\
\leq & \frac{B\left(K_{N}\right)}{N}\left(\left|\psi\left(w_{N}\right)-\Psi_{K_{N}}\left(w_{N}\right)\right|+R(N)\right)+\left|\psi(w)-\psi\left(w_{N}\right)\right|+\frac{B\left(k_{0}\right)}{N} \\
\leq & \frac{B\left(K_{N}\right)}{N} \sup _{x \in[0,1]}\left|\psi(x)-\Psi_{K_{N}}(x)\right|+\left|\psi(w)-\psi\left(w_{N}\right)\right|+\frac{B\left(k_{0}\right)}{N} \\
& +O\left(\frac{f^{-1}\left(K_{N}\right)}{N\left(\log f^{-1}\left(K_{N}\right)\right)^{2}\left(\log f-1\left(K_{N}\right)-1\right)}\right) \\
& +O\left(\frac{\log f^{-1}\left(K_{N}\right)}{N\left(\log f^{-1}\left(K_{N}\right)-1\right)} .\right.
\end{aligned}
$$


We can rewrite (13) to the form

$$
\begin{array}{r}
|(\underline{13})| \leq \frac{B\left(K_{N}\right)}{N}\left(\left|\min \left(\frac{B\left(K_{N}+x\right)}{B\left(K_{N}\right)}, \frac{B\left(K_{N}+w_{N}\right)}{B\left(K_{N}\right)}\right)-\min \left(\psi(x), \psi\left(w_{N}\right)\right)\right|+\right. \\
\left.\left|\min \left(\psi(x), \psi\left(w_{N}\right)\right)-\min (\psi(x), \psi(w))\right|\right) .
\end{array}
$$

If $x<w_{N}$, then $B\left(K_{N}+x\right)<B\left(K_{N}+w_{N}\right)$, then $\psi(x)<\psi\left(w_{N}\right)$ and then

$$
\begin{array}{r}
\left|\min \left(\frac{B\left(K_{N}+x\right)}{B\left(K_{N}\right)}, \frac{B\left(K_{N}+w_{N}\right)}{B\left(K_{N}\right)}\right)-\min \left(\psi(x), \psi\left(w_{N}\right)\right)\right|= \\
\left|\Psi_{K_{N}}(x)-\psi(x)\right| \leq \sup _{x \in[0,1]}\left|\Psi_{K_{N}}(x)-\psi(x)\right| .
\end{array}
$$

If $x<w_{N}$ and $x<w$, then $\min \left(\psi(x), \psi\left(w_{N}\right)\right)-\min (\psi(x), \psi(w))=0$. If $w<x<w_{N}$, then $\left|\min \left(\psi(x), \psi\left(w_{N}\right)\right)-\min (\psi(x), \psi(w))\right|=|\psi(x)-\psi(w)| \leq$ $\left|\psi\left(w_{N}\right)-\psi(w)\right|$. Similarly, in all other cases. Thus we have

$$
|(\underline{13})| \leq \frac{B\left(K_{N}\right)}{N}\left(\sup _{x \in[0,1]}\left|\Psi_{K_{N}}(x)-\psi(x)\right|+\left|\psi\left(w_{N}\right)-\psi(w)\right|\right) .
$$

By (19) we obtain

$$
\begin{aligned}
|(\underline{14})| \leq & \frac{B\left(K_{N}\right)}{N}(\psi(1)-1)\left(\left|\psi(w)-\psi\left(w_{N}\right)\right|+\left|\psi\left(w_{N}\right)-\frac{N}{B\left(K_{N}\right)}\right|\right) \\
= & \frac{B\left(K_{N}\right)}{N}(\psi(1)-1)\left(\left|\psi(w)-\psi\left(w_{N}\right)\right|+\left|\psi\left(w_{N}\right)-\Psi_{K_{N}}\left(w_{N}\right)\right|+R(N)\right) \\
\leq & \frac{B\left(K_{N}\right)}{N}(\psi(1)-1)\left(\left|\psi(w)-\psi\left(w_{N}\right)\right|+\sup _{x \in[0,1]}\left|\Psi_{K_{N}}(x)-\psi(x)\right|+\right. \\
\leq & \left.O\left(\frac{1}{\left(\log f^{-1}\left(K_{N}\right)\right)^{2}}\right)+O\left(\frac{\log f^{-1}\left(K_{N}\right)}{f^{-1}\left(K_{N}\right)}\right)\right) \\
& +O\left(\frac{\left.\sup _{N}\left|\Psi_{K_{N}}(x)-\psi(x)\right|\right) \frac{B\left(K_{N}\right)}{N}}{N\left(\log f^{-1}\left(K_{N}\right)\right)^{2}\left(\log f^{-1}\left(K_{N}\right)-1\right)}\right) \\
& +O\left(\frac{(\psi(1)-1) \log f^{-1}\left(K_{N}\right)}{N\left(\log f^{-1}\left(K_{N}\right)-1\right)}\right)
\end{aligned}
$$


Combining (10), (15), (20), (21), and (22), we have

$$
\begin{aligned}
& \left|F_{N}(x)-g_{w}(x)\right| \\
& \leq \frac{2}{N} \sum_{k=k_{0}}^{K_{N}-1} B(k) \sup _{x \in[0,1]}\left|\Psi_{k}(x)-\psi(x)\right|+\frac{B\left(K_{N}\right)}{N} \sup _{x \in[0,1]}\left|\Psi_{K_{N}}(x)-\psi(x)\right| \\
& +\left|\psi(w)-\psi\left(w_{N}\right)\right|+\frac{B\left(k_{0}\right)}{N}+O\left(\frac{f^{-1}\left(K_{N}\right)}{N\left(\log f^{-1}\left(K_{N}\right)\right)^{2}\left(\log f^{-1}\left(K_{N}\right)-1\right)}\right) \\
& +O\left(\frac{\log f^{-1}\left(K_{N}\right)}{N\left(\log f^{-1}\left(K_{N}\right)-1\right)}\right) \\
& +\frac{B\left(K_{N}\right)}{N}\left(\sup _{x \in[0,1]}\left|\Psi_{K_{N}}(x)-\psi(x)\right|+\left|\psi\left(w_{N}\right)-\psi(w)\right|\right) \\
& +(\psi(1)-1) \frac{B\left(K_{N}\right)}{N}\left(\left|\psi(w)-\psi\left(w_{N}\right)\right|+\sup _{x \in[0,1]}\left|\Psi_{K_{N}}(x)-\psi(x)\right|\right) \\
& +O\left(\frac{(\psi(1)-1) f^{-1}\left(K_{N}\right)}{N\left(\log f^{-1}\left(K_{N}\right)\right)^{2}\left(\log f^{-1}\left(K_{N}\right)-1\right)}\right)+O\left(\frac{(\psi(1)-1) \log f^{-1}\left(K_{N}\right)}{N\left(\log f^{-1}\left(K_{N}\right)-1\right)}\right) \\
& +O\left(\frac{1}{N} \sum_{k=k_{0}+1}^{K_{N}+1} \frac{f^{-1}(k)}{\left(\log f^{-1}(k)\right)^{3}}\right)+O\left(\frac{f^{-1}\left(k_{0}\right)+m}{N}\right) \\
& \leq \frac{2}{N} \sum_{k=k_{0}}^{K_{N}-1} B(k) \sup _{x \in[0,1]}\left|\Psi_{k}(x)-\psi(x)\right|+(\psi(1)+1) \frac{B\left(K_{N}\right)}{N} \sup _{x \in[0,1]}\left|\Psi_{K_{N}}(x)-\psi(x)\right| \\
& +\left(\psi(1) \frac{B\left(K_{N}\right)}{N}+1\right)\left|\psi(w)-\psi\left(w_{N}\right)\right|+\frac{B\left(k_{0}\right)}{N} \\
& +O\left(\frac{\psi(1) B\left(K_{N}\right)}{N\left(\log f^{-1}\left(K_{N}\right)\right)^{2}}\right)+O\left(\frac{1}{N} \sum_{k=k_{0}+1}^{K_{N}+1} \frac{f^{-1}(k)}{\left(\log f^{-1}(k)\right)^{3}}\right) \\
& +O\left(\frac{f^{-1}\left(k_{0}\right)+m}{N}\right)+O\left(\frac{\psi(1) \log f^{-1}\left(K_{N}\right)}{N\left(\log f^{-1}\left(K_{N}\right)-1\right)}\right) .
\end{aligned}
$$

This proves the theorem.

From Theorem [1, we deduce the following corollaries. 


\section{YUKIO OHKUBO — OTO STRAUCH}

Corollary 1. Suppose $f$ is a strictly increasing differentiable function on $\left[p_{m+1}, \infty\right)$ for some integer $m \geq 0$, and satisfies

$$
\lim _{t \rightarrow \infty} t f^{\prime}(t)=\rho>0 .
$$

Let $0<w \leq 1$ and $N_{i}=\pi\left(f^{-1}(i+w)\right)$. Then

$$
\lim _{i \rightarrow \infty}\left\{f\left(p_{N_{i}}\right)\right\}=w
$$

and

$$
\lim _{i \rightarrow \infty} D_{N_{i}}^{*}\left(f\left(p_{m+n}\right), g_{w}\right)=0
$$

where

$$
g_{w}(x)=\frac{1}{e^{\frac{w}{\rho}}} \frac{e^{\frac{x}{\rho}}-1}{e^{\frac{1}{\rho}}-1}+\frac{\min \left(e^{\frac{x}{\rho}}, e^{\frac{w}{\rho}}\right)-1}{e^{\frac{w}{\rho}}} \quad(0 \leq x \leq 1) .
$$

Proof. Let $0<w \leq 1, K_{N_{i}}=\left\lfloor f\left(p_{N_{i}}\right)\right\rfloor, w_{N_{i}}=\left\{f\left(p_{N_{i}}\right)\right\}$. By the prime number theorem, we have

$$
\begin{aligned}
& \pi\left(f^{-1}(i+w)\right)-\pi\left(f^{-1}(i)\right) \\
& =\frac{f^{-1}(i+w)}{\log f^{-1}(i+w)}-\frac{f^{-1}(i)}{\log f^{-1}(i)}+O\left(\frac{f^{-1}(i+w)}{\log ^{2} f^{-1}(i+w)}\right) \\
& =\frac{f^{-1}(i+w)}{\log f^{-1}(i+w)}\left\{1-\frac{f^{-1}(i)}{f^{-1}(i+w)} \frac{\log f^{-1}(i+w)}{\log f^{-1}(i)}+O\left(\frac{1}{\log f^{-1}(i+w)}\right)\right\} .
\end{aligned}
$$

By the assumption, we obtain

$$
\lim _{x \rightarrow \infty} f^{-1}(x)=\infty, \quad \text { and so } \quad \lim _{i \rightarrow \infty} \frac{f^{-1}(i+w)}{\log f^{-1}(i+w)}=\infty .
$$

By Lemma 4, we have $\lim _{i \rightarrow \infty} \frac{f^{-1}(i+w)}{f^{-1}(i)}=e^{\frac{w}{\rho}}>1$. Hence

$$
\lim _{i \rightarrow \infty} \frac{\log f^{-1}(i+w)}{\log f^{-1}(i)}=\lim _{i \rightarrow \infty}\left(\frac{1}{\log f^{-1}(i)} \log \frac{f^{-1}(i+w)}{f^{-1}(i)}\right)+1=1 .
$$

Therefore, (24) and (25) yield

$$
\lim _{i \rightarrow \infty} \pi\left(f^{-1}(i+w)\right)-\pi\left(f^{-1}(i)\right)=\infty .
$$

Hence, there exists $i_{0}$ such that $\pi\left(f^{-1}(i+w)\right)-\pi\left(f^{-1}(i)\right) \geq 1$ for $i \geq i_{0}$. Thus, for $i \geq i_{0}$

$$
f^{-1}(i) \leq p_{N_{i}}<f^{-1}(i+w) \leq p_{N_{i}+1}, \quad \text { so } \quad i \leq f\left(p_{N_{i}}\right)<i+w \leq f\left(p_{N_{i}+1}\right),
$$

and so

$$
K_{N_{i}}=\left\lfloor f\left(p_{N_{i}}\right)\right\rfloor=i .
$$


Successively, we obtain

$$
\begin{aligned}
w_{N_{i}} & =\left\{f\left(p_{N_{i}}\right)\right\}=f\left(p_{N_{i}}\right)-i<w, \\
w & >w_{N_{i}}=w_{N_{i}}-w+w=f\left(p_{N_{i}}\right)-(i+w)+w \geq f\left(p_{N_{i}}\right)-f\left(p_{N_{i}+1}\right)+w, \\
0 & <w-w_{N_{i}} \leq f\left(p_{N_{i}+1}\right)-f\left(p_{N_{i}}\right) .
\end{aligned}
$$

It is shown that

$$
p_{n+1}-p_{n}=O\left(p_{n}^{\theta}\right)
$$

for some $0<\theta<1$, namely, $p_{n+1}-p_{n}=O\left(p_{n}^{0.525}\right)$ due to R. C. B aker, G. H a r m a n and J.P in t z [1]. Hence, by the assumption (23)

$$
f\left(p_{N_{i}+1}\right)-f\left(p_{N_{i}}\right)=\left(p_{N_{i}+1}-p_{N_{i}}\right) f^{\prime}(\xi)=O\left(p_{N_{i}}^{\theta-1}\right), \quad p_{N_{i}} \leq \xi \leq p_{N i+1} .
$$

Therefore, by (27) we have

$$
w_{N_{i}} \rightarrow w \quad(i \rightarrow \infty)
$$

Applying Theorem 1 with $\psi(x)=e^{\frac{x}{\rho}}$, we have

$$
\begin{aligned}
& D_{N_{i}}^{*}\left(f\left(p_{m+n}\right), g_{w}\right) \\
& \leq \frac{2}{N_{i}} \sum_{k=k_{0}}^{i-1} B(k) \sup _{x \in[0,1]}\left|\frac{B(k+x)}{B(k)}-e^{\frac{x}{\rho}}\right|+\left(e^{\frac{1}{\rho}}+1\right) \frac{B(i)}{N_{i}} \sup _{x \in[0,1]}\left|\frac{B(i+x)}{B(i)}-e^{\frac{x}{\rho}}\right| \\
& +\left(e^{\frac{1}{\rho}} \frac{B(i)}{N_{i}}+1\right)\left|e^{\frac{w}{\rho}}-e^{\frac{w_{N}}{\rho}}\right|+\frac{B\left(k_{0}\right)}{N_{i}}+O\left(\frac{1}{N_{i}} \sum_{k=k_{0}+1}^{i+1} \frac{f^{-1}(k)}{\left(\log f^{-1}(k)\right)^{3}}\right) \\
& +O\left(\frac{e^{\frac{1}{\rho}} B(i)}{N_{i}\left(\log f^{-1}(i)\right)^{2}}\right)+\frac{O\left(f^{-1}\left(k_{0}+m\right)\right)}{N_{i}}+O\left(\frac{e^{\frac{1}{\rho}} \log f^{-1}(i)}{N_{i}\left(\log f^{-1}(i)-1\right)}\right) .
\end{aligned}
$$

We note that

$$
\begin{aligned}
& \frac{1}{N_{i}} \sum_{k=k_{0}}^{i-1} B(k)\left|\frac{B(k+x)}{B(k)}-e^{\frac{x}{\rho}}\right|=\frac{1}{N_{i}} \sum_{k=k_{0}}^{i-1}(B(k+1)-B(k)) \times \\
& \frac{\sup _{x \in[0,1]}\left|\frac{B(k+x)}{B(k)}-e^{\frac{x}{\rho}}\right|}{\frac{B(k+1)}{B(k)}-1} .
\end{aligned}
$$

By Lemma 5 we have

$$
\lim _{k \rightarrow \infty} \sup _{x \in[0,1]}\left|\frac{B(k+x)}{B(k)}-e^{\frac{x}{\rho}}\right|=0 .
$$

Combining this with $\lim _{k \rightarrow \infty} \frac{B(k+1)}{B(k)}=\psi(1)=e^{\frac{1}{\rho}}>1$, we get

$$
\lim _{k \rightarrow \infty} \frac{\sup _{x \in[0,1]}\left|\frac{B(k+x)}{B(k)}-e^{\frac{x}{\rho}}\right|}{\frac{B(k+1)}{B(k)}-1}=0 .
$$


By Lemma 5 and (29) we have

$$
\begin{aligned}
& \left|\frac{B\left(K_{N_{i}}+w_{N_{i}}\right)}{B\left(K_{N_{i}}\right)}-e^{\frac{w}{\rho}}\right| \leq\left|\frac{B\left(K_{N_{i}}+w_{N_{i}}\right)}{B\left(K_{N_{i}}\right)}-e^{\frac{w_{N_{i}}}{\rho}}\right|+\left|e^{\frac{w_{N_{i}}}{\rho}}-e^{\frac{w}{\rho}}\right| \\
& \leq \sup _{0 \leq x \leq 1}\left|\frac{B\left(K_{N_{i}}+x\right)}{B\left(K_{N_{i}}\right)}-e^{\frac{x}{\rho}}\right|+\left|e^{\frac{w_{N_{i}}}{\rho}}-e^{\frac{w}{\rho}}\right| \rightarrow 0 \quad(i \rightarrow \infty),
\end{aligned}
$$

so we have

$$
\lim _{i \rightarrow \infty} \frac{B\left(K_{N_{i}}+w_{N_{i}}\right)}{B\left(K_{N_{i}}\right)}=e^{\frac{w}{\rho}} .
$$

Therefore, by (18) and (19) we have

$$
\lim _{i \rightarrow \infty} \frac{B(i)}{N_{i}}=\frac{1}{e^{\frac{w}{\rho}}}
$$

By (31), (32) and (34), we have

$$
\lim _{i \rightarrow \infty} \frac{1}{N_{i}} \sum_{k=0}^{i-1} B(k) \sup _{x \in[0,1]}\left|\Psi_{k}(x)-e^{\frac{x}{\rho}}\right|=0 .
$$

Lastly, we show that $\frac{1}{N_{i}} \sum_{k=k_{0}+1}^{i+1} \frac{f^{-1}(k)}{\left(\log f^{-1}(k)\right)^{3}} \rightarrow 0$. By Cauchy-Stolz lemma, it suffices to prove that

$$
\lim _{i \rightarrow \infty} \frac{\left(\log f^{-1}(i+1)\right)^{3}\left(N_{i}-N_{i-1}\right)}{f^{-1}(i+1)}=\infty .
$$

By (17) we have

$$
N_{i}-N_{i-1}=B\left(i+w_{N_{i}}\right)-B\left(i-1+w_{N_{i-1}}\right)+O\left(\frac{f^{-1}\left(i+w_{N_{i}}\right)}{\left(\log f^{-1}\left(i+w_{N_{i}}\right)\right)^{3}}\right) \text {. }
$$

Since

$$
\begin{aligned}
& f^{-1}\left(i+w_{N_{i}}\right)\left(\log f^{-1}\left(i-1+w_{N_{i-1}}\right)-1\right) \\
& \quad-f^{-1}\left(i-1+w_{N_{i-1}}\right)\left(\log f^{-1}\left(i+w_{N_{i}}\right)-1\right) \\
& =f^{-1}\left(i+w_{N_{i}}\right) \log \frac{f^{-1}\left(i-1+w_{N_{i-1}}\right)}{f^{-1}\left(i+w_{N_{i}}\right)} \\
& \quad+\left\{f^{-1}\left(i+w_{N_{i}}\right)-f^{-1}\left(i-1+w_{N_{i-1}}\right)\right\}\left\{\log f^{-1}\left(i+w_{N_{i}}\right)-1\right\} \\
& \geq f^{-1}(i) \log \frac{f^{-1}\left(i-1+w_{N_{i-1}}\right)}{f^{-1}\left(i+w_{N_{i}}\right)}
\end{aligned}
$$

we obtain

$$
B\left(i+w_{N_{i}}\right)-B\left(i-1+w_{N_{i-1}}\right) \geq \frac{f^{-1}(i) \log \frac{f^{-1}\left(i-1+w_{N_{i-1}}\right)}{f^{-1}\left(i+w_{N_{i}}\right)}}{\left(\log f^{-1}(i)-1\right)\left(\log f^{-1}(i+1)-1\right)} .
$$


Therefore,

$$
\begin{aligned}
& \frac{\left(\log f^{-1}(i)\right)^{3}\left(B\left(i+w_{N_{i}}\right)-B\left(i-1+w_{N_{i-1}}\right)\right)}{f^{-1}(i)} \\
& \geq \frac{\left(\log f^{-1}(i)\right)^{3} \log \frac{f^{-1}\left(i-1+w_{N_{i-1}}\right)}{f^{-1}\left(i+w_{N_{i}}\right)}}{\left(\log f^{-1}(i)-1\right)\left(\log f^{-1}(i+1)-1\right)} \\
& \geq\left(\log f^{-1}(i)\right) \frac{1}{1-\frac{1}{\log f^{-1}(i)}} \frac{1}{\frac{\log f^{-1}(i+1)}{\log f^{-1}(i)}-\frac{1}{\log f^{-1}(i)}} \log \frac{f^{-1}\left(i-1+w_{N_{i-1}}\right)}{f^{-1}\left(i+w_{N_{i}}\right)} \text {. }
\end{aligned}
$$

By Lemma 4 and (29), we have

$$
\lim _{i \rightarrow \infty} \frac{f^{-1}\left(i+w_{N_{i}}\right)}{f^{-1}(i)}=\psi(w)
$$

SO

$$
\frac{f^{-1}\left(i-1+w_{N_{i-1}}\right)}{f^{-1}\left(i+w_{N_{i}}\right)}=\frac{\frac{f^{-1}\left(i-1+w_{N_{i-1}}\right)}{f^{-1}(i-1)}}{\frac{f^{-1}\left(i+w_{N_{i}}\right)}{f^{-1}(i)} \cdot \frac{f^{-1}(i)}{f^{-1}(i-1)}} \rightarrow \frac{\psi(w)}{\psi(w) \psi(1)}=\frac{1}{\psi(1)} \quad(i \rightarrow \infty) .
$$

From (25), (37), and (39), it follows that

$$
\lim _{i \rightarrow \infty} \frac{\left(\log f^{-1}(i)\right)^{3}\left(B\left(i+w_{N_{i}}\right)-B\left(i-1+w_{N_{i-1}}\right)\right)}{f^{-1}(i)}=\infty .
$$

By (25) and (38) we have

$$
\lim _{i \rightarrow \infty} \frac{\log f^{-1}(i)}{\log f^{-1}\left(i+w_{N_{i}}\right)}=1 .
$$

Hence, by (36), (40), and (41) we have

$$
\begin{aligned}
& \frac{\left(\log f^{-1}(i)\right)^{3}\left(N_{i}-N_{i-1}\right)}{f^{-1}(i)}= \\
& \quad \frac{\left(\log f^{-1}(i)\right)^{3}\left(B\left(i+w_{N_{i}}\right)-B\left(i-1+w_{N_{i-1}}\right)\right)}{f^{-1}(i)}+ \\
& O\left(\frac{f^{-1}\left(i+w_{N_{i}}\right)}{f^{-1}(i)} \cdot\left(\frac{\log f^{-1}(i)}{\log f^{-1}\left(i+w_{N_{i}}\right)}\right)^{3}\right) \\
& \quad \text { as } \quad i \rightarrow \infty .
\end{aligned}
$$




\section{YUKIO OHKUBO - OTO STRAUCH}

From (42),

$$
\lim _{i \rightarrow \infty} f^{-1}(i) / f^{-1}(i+1)=1 / e^{1 / \rho}, \quad \text { and } \quad \lim _{i \rightarrow \infty} \log f^{-1}(i+1) / \log f^{-1}(i)=1,
$$

we arrive at (35). Consequently, the right hand side of (30) tends to 0 as $i \rightarrow \infty$. Thus $\lim _{i \rightarrow \infty} D_{N_{i}}^{*}\left(f\left(p_{m+n}\right) \bmod 1, g_{w}\right)=0$.

CorollaRy 2. Let $r>0,0<w \leq 1$, let $N_{i}=\pi\left(b^{\frac{i+w}{r}}\right), i=1,2, \ldots$, and let

Then we have

$$
g_{w}(x)=\frac{1}{b^{\frac{w}{r}}} \frac{b^{\frac{x}{r}}-1}{b^{\frac{1}{r}}-1}+\frac{\min \left(b^{\frac{x}{r}}, b^{\frac{w}{r}}\right)-1}{b^{\frac{w}{r}}} \quad(0 \leq x \leq 1) .
$$

$$
\begin{aligned}
D_{N_{i}}^{*}\left(\log _{b} p_{n}^{r}, g_{w}\right) & \leq b^{\frac{1}{r}}(\log b)\left(2+\left(b^{\frac{1}{r}}+1\right) \frac{\log b}{r}\right) \frac{\log p_{N_{i}}}{\left(\log p_{N_{i}}-\left(1+\frac{1}{r}\right) \log b\right)^{2}} \\
& +C_{1}\left(\frac{b^{\frac{2}{r}} r}{\log b}+b^{\frac{1}{r}} \log b\right) \frac{\log p_{N_{i}}}{\left(\log p_{N_{i}}-\left(1+\frac{1}{r}\right) \log b\right)^{3}} \\
& +C_{2}\left(b^{2}+\frac{b^{2}}{2 \log b-1}\right) b^{\frac{2}{r}} r \frac{\log p_{N_{i}}}{p_{N_{i}}^{1-\theta}} \\
& +C_{3} b^{\frac{2}{r}} r \frac{\left(\log p_{N_{i}}\right)^{2}+\log p_{N_{i}} \log \log p_{N_{i}}}{p_{N_{i}}\left(r \log p_{N_{i}}-r-\log b\right)}
\end{aligned}
$$

where $N_{i} \geq 6$ for $i=1,2, \ldots$ and $C_{j}$ is a constant for $j=1,2,3$.

P r o o f. Applying Theorem 1 and Corollary 1 with

- $f(t)=\log _{b} t^{r}$ for $t \geq 1$,

- $m=0$,

- $k_{0}=\lceil 2 r\rceil$,

- $f^{-1}(t)=b^{\frac{t}{r}}$ for $t \geq 0$,

- $\rho=\lim _{t \rightarrow \infty} t f^{\prime}(t)=\frac{r}{\log b}$,

- $\psi(x)=b^{\frac{x}{r}}$,

- $B(x)=\frac{r b^{\frac{x}{r}}}{x \log b-r}$,

- $N_{i}=\pi\left(b^{\frac{i+w}{r}}\right)$,

- $K_{N_{i}}=\left\lfloor r \log _{b} p_{N_{i}}\right\rfloor=i$ by (26),$w_{N_{i}}=\left\{r \log _{b} p_{N_{i}}\right\}$, 
we have

$$
\begin{aligned}
& D_{N_{i}}^{*}\left(\log _{b} p_{n}^{r}, g_{w}\right) \leq \frac{2 r}{N_{i}} \sum_{2 r \leq k \leq i-1} \frac{b^{\frac{k}{r}}}{k-r} \sup _{x \in[0,1]}\left|b^{\frac{x}{r}}\left(\frac{k-r}{k+x-r}-1\right)\right| \\
& +\frac{\left(b^{\frac{1}{r}}+1\right) r b^{\frac{i}{r}}}{(i-r) N_{i}} \sup _{x \in[0,1]}\left|b^{\frac{x}{r}}\left(\frac{i-r}{i+x-r}-1\right)\right|+\left(\frac{r b^{\frac{i+1}{r}}}{N_{i}}+1\right)\left|b^{\frac{w}{r}}-b^{\frac{w_{N}}{r}}\right| \\
& +\frac{b^{2+\frac{1}{r}}}{(2 \log b-1) N_{i}}+O\left(\frac{r^{3}}{(\log b)^{3} N_{i}} \sum_{2 r+1 \leq k \leq i+1} \frac{b^{\frac{k}{r}}}{k^{3}}\right)+O\left(\frac{r^{3} b^{\frac{i+1}{r}}}{(\log b)^{2} i^{2}(i-r) N_{i}}\right) \\
& +O\left(\frac{b^{2+\frac{1}{r}}}{N_{i}}\right)+O\left(\frac{b^{\frac{1}{r}}(\log b) i}{N_{i}(i \log b-r)}\right) .
\end{aligned}
$$

Since

$$
\sup _{x \in[0,1]}\left|b^{\frac{x}{r}}\left(\frac{k-r}{k+x-r}-1\right)\right|<\frac{b^{\frac{1}{r}}}{k-r}
$$

and that $\frac{b^{\frac{x}{r}}}{(x-r)^{2}}$ is increasing for $x \geq 2 r$, we have

$$
\sum_{2 r \leq k \leq i-1} \frac{b^{\frac{k}{r}}}{k-r} \sup _{x \in[0,1]}\left|b^{\frac{x}{r}}\left(\frac{k-r}{k+x-r}-1\right)\right| \leq b^{\frac{1}{r}} \int_{2 r}^{i} \frac{b^{\frac{x}{r}}}{(x-r)^{2}} \mathrm{~d} x .
$$

Using the integration by parts, we have

$$
\begin{aligned}
\int_{2 r}^{i} \frac{b^{\frac{x}{r}}}{(x-r)^{2}} \mathrm{~d} x \leq & \frac{r b^{\frac{i}{r}}}{(\log b)(i-r)^{2}}+2 \frac{r}{\log b} \int_{2 r}^{i} \frac{b^{\frac{x}{r}}}{(x-r)^{3}} \mathrm{~d} x \\
\leq & \frac{r b^{\frac{i}{r}}}{(\log b)(i-r)^{2}} \\
& +2 \frac{r}{\log b}\left\{\frac{r b^{\frac{i}{r}}}{(\log b)(i-r)^{3}}+3 \frac{r}{\log b} \int_{2 r}^{i} \frac{b^{\frac{x}{r}}}{(x-r)^{4}} \mathrm{~d} x\right\} \\
\leq & \frac{r b^{\frac{i}{r}}}{(\log b)(i-r)^{2}}+\frac{8 r^{2} b^{\frac{i}{r}}}{(\log b)^{2}(i-r)^{3}},
\end{aligned}
$$

because $\frac{e^{\frac{x}{r}}}{(x-r)^{4}}$ is increasing for $x \geq 2 r$. Hence

$$
\begin{array}{r}
\sum_{2 r \leq k<i} \frac{b^{\frac{k}{r}}}{k-r} \sup _{x \in[0,1]}\left|b^{\frac{x}{r}}\left(\frac{k-r}{k+x-r}-1\right)\right| \\
\leq \frac{r b^{\frac{i+1}{r}}}{(\log b)^{2}(i-r)^{2}}\left(\log b+\frac{8 r}{i-r}\right) .
\end{array}
$$




\section{YUKIO OHKUBO - OTO STRAUCH}

Similarly, we have

$$
\sum_{2 r+1 \leq k \leq i+1} \frac{b^{\frac{k}{r}}}{k^{3}} \leq \frac{4 r b^{\frac{i+2}{r}}}{(\log b)(i+2)^{3}} .
$$

By (27), we have for $w \in[0,1)$,

$$
\begin{aligned}
\left|b^{\frac{w}{r}}-b^{\frac{w_{N_{i}}}{r}}\right| & \leq \frac{b^{\frac{1}{r}} \log b}{r}\left(f\left(p_{N_{i}+1}\right)-f\left(p_{N_{i}}\right)\right) \\
& \leq b^{\frac{1}{r}}\left(\frac{p_{N_{i}+1}-p_{N_{i}}}{p_{N_{i}}}\right) .
\end{aligned}
$$

By (28) and (48), we obtain

$$
\left|b^{\frac{u}{r}}-b^{\frac{w_{N_{i}}}{r}}\right|=O\left(\frac{b^{\frac{1}{r}}}{p_{N_{i}}^{1-\theta}}\right)=O\left(\frac{b^{\frac{1}{r}}}{N_{i}^{1-\theta}\left(\log N_{i}\right)^{1-\theta}}\right),
$$

where $\theta=0.525$.

By (44), (45), (46), (47), and (49), we obtain

$$
\begin{aligned}
D_{N_{i}}^{*}\left(\log _{b} p_{n}^{r}, g_{w}\right) \leq & \left(\frac{2}{\log b} b^{\frac{1}{r}} r^{2}+\left(b^{\frac{2}{r}}+b^{\frac{1}{r}}\right) r\right) \frac{b^{\frac{i}{r}}}{(i-r)^{2} N_{i}} \\
& +\frac{16 b^{\frac{1}{r}} r^{3}}{(\log b)^{2}} \frac{b^{\frac{i}{r}}}{(i-r)^{3} N_{i}} \\
& +\operatorname{const} b^{\frac{2}{r}} r \frac{b^{\frac{i}{r}}}{N_{i}^{2-\theta}\left(\log N_{i}\right)^{1-\theta}}+\operatorname{const} b^{\frac{1}{r}} \frac{1}{N_{i}^{1-\theta}\left(\log N_{i}\right)^{1-\theta}} \\
& +\frac{b^{2+\frac{1}{r}}}{2 \log b-1} \frac{1}{N_{i}} \\
& +\operatorname{const} \frac{b^{\frac{2}{r}} r^{4}}{(\log b)^{4}} \frac{b^{\frac{i}{r}}}{i^{3} N_{i}}+\operatorname{const} \frac{b^{\frac{1}{r}} r^{3}}{(\log b)^{2}} \frac{b^{\frac{i}{r}}}{i^{2}(i-r) N_{i}} \\
& +\operatorname{const} b^{2+\frac{1}{r}} \frac{1}{N_{i}}+\operatorname{const}(\log b) b^{\frac{1}{r}} \frac{i}{N_{i}(i \log b-r)}
\end{aligned}
$$

where each "const" means a positive constant. Since

$$
\begin{gathered}
r \log _{b} p_{N_{i}}=i+w_{N_{i}} \leq i+1, \\
i \geq \frac{r}{\log b} \log p_{N_{i}}-1 .
\end{gathered}
$$




\section{DISTRIBUTION OF LEADING DIGITS OF NUMBERS II}

Since

and that

$$
i<i+w_{N_{i}}=\frac{r}{\log b} \log p_{N_{i}}
$$

$$
p_{n}<n(\log n+\log \log n) \quad \text { for } n \geq 6
$$

by J. B. R os ser and L. S choenfeld [10, we have

and so

$$
i<\frac{r}{\log b}\left(\log N_{i}+\log \left(\log N_{i}+\log \log N_{i}\right)\right),
$$

$$
b^{\frac{i}{r}}<N_{i}\left(\log N_{i}+\log \log N_{i}\right)
$$

for $N_{i} \geq 6$. By (51), (53), (54), and

$$
n \log n<p_{n} \quad \text { for } n \geq 1,
$$

by J. B. Rosser and L.Schoenfeld [10], we have

$$
\begin{aligned}
\frac{b^{\frac{i}{r}}}{(i-r)^{2} N_{i}} & <\frac{\log \left(N_{i} \log N_{i}\right)}{\left(\frac{r}{\log b} \log p_{N_{i}}-r-1\right)^{2}}<\frac{\log p_{N_{i}}}{\left(\frac{r}{\log b} \log p_{N_{i}}-r-1\right)^{2}}, \\
\frac{b^{\frac{i}{r}}}{(i-r)^{3} N_{i}} & <\frac{\log \left(N_{i} \log N_{i}\right)}{\left(\frac{r}{\log b} \log p_{N_{i}}-r-1\right)^{3}}<\frac{\log p_{N_{i}}}{\left(\frac{r}{\log b} \log p_{N_{i}}-r-1\right)^{3}}, \\
\frac{b^{\frac{i}{r}}}{N_{i}^{2-\theta}\left(\log N_{i}\right)^{1-\theta}} & <\frac{\log \left(N_{i} \log N_{i}\right)}{N_{i}^{1-\theta}\left(\log N_{i}\right)^{1-\theta}}, \\
\frac{b^{\frac{i}{r}}}{i^{3} N_{i}} & <\frac{\log \left(N_{i} \log N_{i}\right)}{\left(\frac{r}{\log b} \log p_{N_{i}}-1\right)^{3}}<\frac{\log p_{N_{i}}}{\left(\frac{r}{\log b} \log p_{N_{i}}-1\right)^{3}}, \\
\frac{b^{\frac{i}{r}}}{i^{2}(i-r) N_{i}} & <\frac{\log \left(N_{i} \log N_{i}\right)}{\left(\frac{r}{\log b} \log p_{N_{i}}-1\right)^{2}\left(\frac{r}{\log b} \log p_{N_{i}}-r-1\right)} \\
& <\frac{\log p_{N_{i}}}{\left(\frac{r}{\log b} \log p_{N_{i}}-1\right)^{2}\left(\frac{r}{\log b} \log p_{N_{i}}-r-1\right)}, \\
\frac{i}{N_{i}(i-r)} & <\frac{r}{\log b} \frac{\log \left(N_{i} \log \left(N_{i} \log N_{i}\right)\right)}{\left.\frac{r}{\log b} \log p_{N_{i}}-r-1\right)} \\
& <\frac{r}{\log b} \frac{\log p_{N_{i}}}{N_{i}\left(\frac{r}{\log b} \log p_{N_{i}}-r-1\right)}, \\
\frac{1}{N_{i}} & <\frac{1}{\pi\left(b^{\frac{i}{r}}\right)} \sim \frac{\log b}{r} \frac{i}{b^{\frac{i}{r}}}<\frac{\log b}{r} \frac{r}{\left.b^{(\log b} p_{N_{i}}-\frac{1}{r}\right)} \log p_{N_{i}} \\
& =b^{\frac{1}{r} \frac{\log p_{N_{i}}}{p_{N_{i}}}} .
\end{aligned}
$$


Overall, by (150), we have

$$
\begin{aligned}
& D_{N_{i}}^{*}\left(\log _{b} p_{n}^{r}, g_{w}\right) \leq\left(\frac{2 b^{\frac{1}{r}} r^{2}}{\log b}+\left(b^{\frac{2}{r}}+b^{\frac{1}{r}}\right) r\right) \frac{\log p_{N_{i}}}{\left(\frac{r}{\log b} \log p_{N_{i}}-r-1\right)^{2}} \\
& +\frac{16 b^{\frac{1}{r}} r^{3}}{(\log b)^{2}} \frac{\log p_{N_{i}}}{\left(\frac{r}{\log b} \log p_{N_{i}}-r-1\right)^{3}}+\text { const } b^{\frac{2}{r}} r \frac{\log p_{N_{i}}}{p_{N_{i}}^{1-\theta}} \\
& + \text { const } b^{\frac{1}{r}} \frac{1}{p_{N_{i}}^{1-\theta}}+\text { const } \frac{b^{2+\frac{2}{r}}}{2 \log b-1} \frac{\log p_{N_{i}}}{p_{N_{i}}} \\
& + \text { const } \frac{b^{\frac{2}{r}} r^{4}}{(\log b)^{4}} \frac{\log p_{N_{i}}}{\left(\frac{r}{\log b} \log p_{N_{i}}-1\right)^{3}} \\
& + \text { const } \frac{b^{\frac{1}{r}} r^{3}}{(\log b)^{2}} \frac{\log p_{N_{i}}}{\left(\frac{r}{\log b} \log p_{N_{i}}-1\right)^{2}\left(\frac{r}{\log b} \log p_{N_{i}}-r-1\right)} \\
& + \text { const } b^{2+\frac{2}{r}} \frac{\log p_{N_{i}}}{p_{N_{i}}} \\
& + \text { const } b^{\frac{2}{r}} r \frac{\left(\log p_{N_{i}}\right)^{2}+\log p_{N_{i}} \log \log p_{N_{i}}}{p_{N_{i}}\left(r \log p_{N_{i}}-r-\log b\right)} \\
& \leq b^{\frac{1}{r}}(\log b)\left(2+\left(b^{\frac{1}{r}}+1\right) \frac{\log b}{r}\right) \frac{\log p_{N_{i}}}{\left(\log p_{N_{i}}-\left(1+\frac{1}{r}\right) \log b\right)^{2}} \\
& + \text { const }\left(\frac{b^{\frac{2}{r}} r}{\log b}+b^{\frac{1}{r}} \log b\right) \frac{\log p_{N_{i}}}{\left(\log p_{N_{i}}-\left(1+\frac{1}{r}\right) \log b\right)^{3}} \\
& + \text { const }\left(b^{2}+\frac{b^{2}}{2 \log b-1}\right) b^{\frac{2}{r}} r \frac{\log p_{N_{i}}}{p_{N_{i}}^{1-\theta}} \\
& + \text { const } b^{\frac{2}{r}} r \frac{\left(\log p_{N_{i}}\right)^{2}+\log p_{N_{i}} \log \log p_{N_{i}}}{p_{N_{i}}\left(r \log p_{N_{i}}-r-\log b\right)}
\end{aligned}
$$

for $N_{i} \geq 6$.

From Corollary 2 it follows immediately:

Corollary 3. Let $r>0,0<w \leq 1$, let $N_{i}=\pi\left(b^{\frac{i+w}{r}}\right), i=1,2, \ldots$, and let

Then we have

$$
g_{w}(x)=\frac{1}{b^{\frac{w}{r}}} \frac{b^{\frac{x}{r}}-1}{b^{\frac{1}{r}}-1}+\frac{\min \left(b^{\frac{x}{r}}, b^{\frac{w}{r}}\right)-1}{b^{\frac{w}{r}}} \quad(0 \leq x \leq 1) .
$$

$$
\lim _{i \rightarrow \infty}\left(\log p_{N_{i}}\right) D_{N_{i}}^{*}\left(\log _{b} p_{n}^{r}, g_{w}\right) \leq b^{\frac{1}{r}}(\log b)\left(2+\left(b^{\frac{1}{r}}+1\right) \frac{\log b}{r}\right) .
$$




\section{DISTRIBUTION OF LEADING DIGITS OF NUMBERS II}

COROllaRY 4. Let $r$ be a positive integer. Then

where

$$
\lim _{r \rightarrow \infty} r D_{\pi\left(b^{r}\right)}^{*}\left(\log _{b} p_{n}^{r}, g_{1}\right) \leq 2,
$$

$$
g_{1}(x)=\frac{b^{\frac{x}{r}}-1}{b^{\frac{1}{r}}-1} \quad(0 \leq x \leq 1)
$$

Proof. Let $r \geq 3$. Put $w=1$ and $i=r^{2}-1$ in Corollary 3. Then $N_{i}=\pi\left(b^{r}\right)$. By (52), we have

and so

$$
\log p_{N_{i}}>\left(r-\frac{1}{r}\right) \log b
$$

$$
\frac{r}{\log p_{N_{i}}} \leq \frac{r}{\left(r-\frac{1}{r}\right) \log b} \rightarrow \frac{1}{\log b}
$$

as $r \rightarrow \infty$. From this and Corollary 3 , the desired inequality follows.

\section{REFERENCES}

[1] BAKER, R. C.-HARMAN, G.-PINTZ, J.: The difference between consecutive primes, II, Proc. London Math. Soc. (3) 83 (2001), no. 3, 532-562.

[2] BINGHAM, N. H.-GOLDIE, C. M.-TEUGELS, J. L.: Regular Variation. In: Encyclopedia of Mathematics and its Applications Vol. 27. Cambridge University Press, Cambridge, 1987.

[3] DIACONIS, P.: The distribution of leading digits and uniform distribution mod 1, Ann. Probability 5 (1977), no. 1, 72-81.

[4] ELIAHOU, S.-MASSÉ, B.-SCHNEIDER, D.: On the m.ntisa distribution of powers of natural and prime numbers, Acta Math. Hungar. 139 (1-2) (2013), 49-63.

[5] INGHAM, A. E.: The Distribution of Prime Numbers, Cambridge Univ. Press, New York, 1932.

[6] KUIPERS, L.-NIEDERREITER, H.: Uniform Distribution of Sequences. In:Pure and Applied Mathematics. Wiley-Interscience [John Wiley \& Sons], New York-London-Sydney, 1974; Reprint: Dover Publications, Inc. Mineola, New York, 2006.

[7] OHKUBO, Y.: On sequences involving primes, Unif. Distrib. Theory 6 (2011), no. 2, 221-238.

[8] OHKUBO, Y.-STRAUCHI, O.: Distribution of leading digits of numbers, Unif. Distrib. Theory 11 (2016), no. 1, 23-45.

[9] PAVLOV, A.I.: On distribution of fractions parts and Benford's law Izv. Akad. Nauk SSSR Ser. Mat. 45 (1981), no. 4, 760-774. (In Russian)

[10] ROSSER, J. B.-SCHOENFELD, L.: Approximate formulas for some functions of prime numbers Illinois J. Math. 6 (1962), 64-94. 


\section{YUKIO OHKUBO - OTO STRAUCH}

[11] SENETA, E.: Regularly Varying Functions. In: Lecture Notes in Math. Vol. 508. Springer-Verlag, Berlin, 1976.

[12] STRAUCH, O.-PORUBSKÝ, Š.: Distribution of Sequences: A Sampler. In: Series of the Slovak Academy of Sciences. Peter Lang, Frankfurt am Main, 2005.

[13] WINTNER, A.: On the cyclical distribution of logarithms of the prime numbers, Quart. J. Math. 6 (1935), no.1, 65-68.

Received November 6, 2017

Accepted May 16, 2018

\section{Yukio Ohkubo}

Department of Business Administration

The International University of Kagoshima

8-34-1 Sakanoue, Kagoshima-shi 891-0197 JAPAN

E-mail: ohkubo@eco.iuk.ac.jp

\section{Oto Strauch}

Mathematical Institute

Slovak Academy of Sciences

Štefánikova 49

SK-814 73 Bratislava

SLOVAKIA

E-mail: strauch@mat.savba.sk 